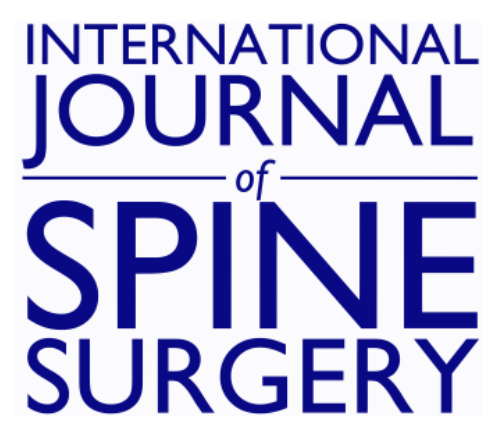

\title{
Resurgence of the Role of Computed-Tomography Myelogram in the Era of MRI for Surgical Decision-Making of Lumbar Degenerative Disc Disorder
}

Keyur Kantilal Akbari, Vigneshwara Badikillaya, Muralidharan Venkatesan, Rochita V. Ramanan and Sajan K. Hegde

Int J Spine Surg 2021, 15 (4) 788-794

doi: https://doi.org/10.14444/8101

http://ijssurgery.com/content/15/4/788

This information is current as of April 25, 2023.

Email Alerts Receive free email-alerts when new articles cite this article. Sign up at:

http://ijssurgery.com/alerts

The International Journal of Spine Surgery

2397 Waterbury Circle, Suite 1,

Aurora, IL 60504, Phone: +1-630-375-1432 


\title{
Resurgence of the Role of Computed-Tomography Myelogram in the Era of MRI for Surgical Decision-Making of Lumbar Degenerative Disc Disorder
}

\author{
KEYUR KANTILAL AKBARI, MS(Orth), VIGNESHWARA BADIKILLAYA, MS(Orth), MURALIDHARAN \\ VENKATESAN, MRCS(Ed), MCh(Orth), FRCS(Tr\&Orth), ROCHITA V. RAMANAN, MD, DNB, DMRD, \\ SAJAN K. HEGDE, MS(Orth) \\ Apollo Hospital, Chennai, India
}

\begin{abstract}
Background: Since the introduction of magnetic resonance imaging (MRI) into clinical practice in the mid-1980s, the role of computed tomography myelography (CTM) has become less important in spinal diagnostics but remains a method that is probably even superior to MRI for special clinical issues. The study aims to report the diagnostic utility of CTM as an adjunct to MRI in lumbar degenerative disc disorder (DDD).

Methods: Included were 20 patients who presented with symptomatic DDD but with MRI findings that did not correlate with the clinical features. These patients underwent CTM as an additional imaging technique to aid preoperative surgical decision-making. Both imaging modalities were compared for the identification of the impinging pathology as well as the number of levels of compression.

Results: MRI revealed compression and/or impingement at 38 levels, whereas CTM revealed these at 29 levels. Of 20 patients, 18 underwent surgery, and a total of 29 levels were decompressed as localized in the CTM. The visual analog scale (VAS) score for back pain and leg pain at baseline were $6 \pm 0.7$ and $7 \pm 0.4$, respectively, and at 6 months postintervention (surgical/conservative) were $2 \pm 0.8$ and $0.3 \pm 0.1$, respectively. The Oswestry Disability Index scores at baseline and 6 months postintervention were $56 \pm 6.9$ and $18 \pm 4.2$, respectively $(P<.0001)$. There was agreement on the number of levels between MRI and CTM in 10 patients (50\%). MRI overestimated the number of involved levels in 9 patients $(45 \%)$, whereas in the remaining 1 patient $(5 \%)$, MRI underestimated the number of involved levels. The weighted $\kappa$ value for agreement between MRI and CTM on the number of levels involved necessitating decompression was $0.4(95 \% \mathrm{CI}, 0.18-0.77 ; P=.0009)$.
\end{abstract}

Conclusions: CTM has a role as an adjunct imaging modality to formulate an effective management plan in patients presenting with symptomatic lumbar DDD in cases where MRI findings are inconclusive and ambiguous.

Level of Evidence: 4.

Lumbar Spine

Keywords: CT myelogram, lumbar canal stenosis, MRI

\section{INTRODUCTION}

Since the introduction of magnetic resonance imaging (MRI) into clinical practice in the mid1980s, the popularity of computed tomography myelogram (CTM) in the clinical practice has showed a decreasing trend. ${ }^{1-4}$ MRI scores over myelography because it is noninvasive with no injection of intrathecal contrast and also has no radiation exposure with better delineation of soft tissues. Despite these advantages, MRI is contraindicated in cases in which the patient has a pacemaker or is claustrophobic, and it may be inadequate due to suboptimal images, as in a previous case of operation with metallic implants in situ. ${ }^{5}$ Obtaining high-quality MRI also depends on the patient's ability to lie still during the image acquisition process, and this is difficult for those with significant pain. Even for a cooperative patient with no contraindication or no previous metal implants, a definitive diagnosis for surgical planning by MRI may not be possible. In 1991 Goldberg et $\mathrm{al}^{6}$ reported that in their series, MRI was not able to clarify the presence of neural compression by disc herniation or nondiscogenic degenerative process in one-third of the patients. It is not uncommon when clinicians face a surgical decision dilemma in patients presenting with symptomatic lumbar degenerative disc disorder (DDD) that MRI findings 
either do not reveal the compressive pathology or are unable to ascertain the offending level when widespread multilevel changes are seen. In such cases, CTM can be used as an adjunct to aid definitive surgical planning.

The purpose of this study is to compare the diagnostic utility of CTM as an adjunct to MRI in patients presenting with symptomatic lumbar DDD.

\section{MATERIALS AND METHODS}

After obtaining approval from the institutional review board, we prospectively reviewed patients who presented to our outpatient clinic between October 2018 and October 2019 with a symptomatic degenerative lumbar disorder and in whom MRI findings were inconclusive (ie, either not revealing the compressive pathology or being unable to ascertain the offending level when widespread multilevel impingement was seen). We excluded patients with a compression fracture of the lumbar spine, tumor, and infection, and patients in whom MRI was not performed due to absolute contraindications.

In our practice MRI is the standard advanced imaging modality for patients presenting with symptomatic lumbar DDD. Those who fail a nonoperative line of management are offered surgical intervention to help alleviate pain and improve their functional ability. CTM is done as an adjunct only in cases in which MRI is ambiguous or inconclusive.

During the study period, 310 patients presented to our outpatient department with symptomatic lumbar DDD, 290 of whom were managed nonoperatively $(70 / 290)$ or surgically $(220 / 290)$ on the basis of MRI findings alone. Only in 20 patients were MRI findings ambiguous and additional imaging CTM was used as an adjunct to help decision-making; these 20 were included in the study. All patients had MRI scans with multiplanar $\mathrm{T} 1$ and $\mathrm{T} 2$ weighted image sequences as the first diagnostic modality, and CTM was performed as a second procedure to obtain more information to aid surgical decision-making.

Patients underwent surgical management or conservative management on the basis of CTM findings. All patients were followed up at 3 and 6 months postintervention (surgical/conservative). $\mathrm{Pa}-$ tient-reported outcome measures visual analog scale $(\mathrm{VAS})^{7}$ for back pain and leg pain and Oswestry
Disability Index (ODI $)^{8}$ were collected at baseline and follow-up.

\section{High-Resolution CT Protocol}

CT examinations were obtained with the Aquilion One CT system (Canon, Tokyo, Japan) on the 64-detector helical mode; $10 \mathrm{~mL}$ of iopromide (Ultravist 370; Schering, Berlin, Germany) was injected into the subarachnoid space via a lumbar puncture to obtain a myelogram. Following injection of contrast, patients were asked to lie supine for 45 minutes for even distribution in the subarachnoid space before the CT scan. The tube voltage was set at $135 \mathrm{kV}$ and tube current at automatic dose modulation. A section thickness of $0.5 \mathrm{~mm}$ with a pitch factor of 0.8 was used.

\section{Image Analysis}

The images were analyzed on a dedicated Vitrea workstation (Vital Images, Minnetonka, MN). Multiplanar reconstructions in the coronal, sagittal, and oblique planes were read in addition to the axial sections by a senior musculoskeletal radiologist with 26 years of experience. Dynamic oblique reconstructions were especially helpful to correct for the obliquity of the spine. Curvilinear reconstructions were also obtained with the center line through the spinal canal. These yielded true sagittal images along the length of the spine in one 2-D plane, giving a panoramic view and avoiding errors in interpretation due to scoliotic curves of the spine.

The spine was studied for disc height maintenance, disc protrusions and their extent, facet-joint abnormalities, ligamentum flavum thickening, central and lateral canal stenosis, neural foraminal stenosis, and compression of the opacified thecal sac.

\section{Surgical Planning}

Both MRI and CTM images were reviewed by the surgical team and analyzed to locate the level of the impingement and number of levels. Compression location and levels according to MRI and CTM were compared. Compression cause was categorized as either disc herniation alone or a combination of disc herniation with nondiscogenic degenerative processes (NDP), which included canal stenosis due to flavum or facetal hypertrophy. The surgical team agreed on the levels of decompression accord- 
Table 1. Levels of compression as per computed tomography myelogram.

\begin{tabular}{lc}
\hline Level of Compression & No. of Cases \\
\hline L1-L2 & 1 \\
L2-L3 & 7 \\
L3-L4 & 11 \\
L4-L5 & 9 \\
L5-S1 & 1 \\
\hline
\end{tabular}

ing to CTM findings in correlation with the patient's clinical findings.

\section{Statistical Calculations}

Data analysis was done using SPSS, version 13 (SPSS Inc, Chicago, IL). ${ }^{9}$ A paired $t$ test was used to compare the difference between preoperative and postoperative VAS and ODI scores. A $P \leq .05$ was considered to indicate a statistically significant difference. The $\kappa$ results of the agreement were interpreted using Landis and Koch's ${ }^{10}$ suggestions: $<.0$ is poor; $.0-.20$ is slight; .20-.40 is fair; .41-.60 is moderate; .61-.80 is substantial, and $.81-1.00$ is almost perfect agreement.

\section{RESULTS}

The mean age of patients in the present cohort was 58.4 years (range, 40-76 years). There were 12 male $(60 \%)$ and 8 female $(40 \%)$ patients.

MRI revealed compression and/or impingement at 38 levels and CTM, at 29 levels. Of 20 patients, 18 underwent surgery and a total of 29 levels were decompressed as diagnosed on CTM and clinical assessment. Two patients were managed conservatively because the CTM did not show any significant compression. Table 1 shows the number of levels of impingement, and Table 2 shows the various pathologies found on CTM. Interobserver reliability between the musculoskeletal radiologist and the surgical team was $100 \%$.

Transforaminal lumbar interbody fusion (TLIF) was the commonest surgical procedure performed in 13 patients $(65 \%)$, followed by laminectomy in 3 patients $(15 \%)$ and posterolateral fusion in 2 patients $(10 \%)$. Two patients $(10 \%)$ were treated conservatively (Table 3 ).

Table 2. Pathology identified by CTM and MRI.

\begin{tabular}{lrr}
\hline & CTM & MRI \\
\hline Disc herniation only & 1 & 0 \\
Disc herniation along with other degenerative disorders & 28 & 38 \\
Total levels & 29 & 38 \\
\hline
\end{tabular}

Abbreviation: CTM, computed tomography myelography.
Table 3. Plan of management of cases.

\begin{tabular}{lc}
\hline Management & Cases \\
\hline TLIF & 13 \\
Laminectomy & 3 \\
Posterolateral fusion & 2 \\
Conservative & 2 \\
\hline
\end{tabular}

Abbreviation: TLIF, transforaminal lumbar interbody fusion.

In the 2 patients for whom surgery was not performed, MRI showed significant disease; however, the CTM did not show any significant compression. The resultant decrease in symptoms at the final follow-up emphasized the accuracy of the CTM.

The mean preoperative VAS scores for back pain and leg pain were $6 \pm 0.73$ and $7 \pm 0.4$, respectively, with significant improvement at final follow-up of 6 months when the VAS scores for back pain and leg pain were $2 \pm 0.80$ and $0.3 \pm 0.1$, respectively. The paired samples $t$ test showed that this improvement was significant $(P<.0001 ; 95 \% \mathrm{CI}, 3 .-4.5)$. The mean preoperative ODI score was $56 \pm 6.92$ and the average postmanagement (surgical/conservative) ODI at 6 months was $18 \pm 4.28$ (Table 4).

A total of 18 patients $(90 \%)$ had no radicular pain at the final follow-up at 6 months, whereas 2 patients $(10 \%)$ who underwent conservative treatment had significant improvement but persistent residual radicular pain. There was agreement on the number of levels between MRI and CT myelogram in 10 cases $(50 \%)$. The MRI overestimated the number of involved levels in 9 cases $(45 \%$; Figure $1)$, whereas in the remaining 1 case $(5 \%)$, MRI underestimated the number of involved levels. For that patient, CTM showed an extruded left paracentral disc compressing the traversing nerve root at the L4-L5 level. A TLIF L4-L5 was done, and at 6 months follow-up the patient had no radicular symptoms (Figure 2).

The weighted $\kappa$ value for agreement between MRI and CTM on the number of levels involved necessitating decompression was moderate at .47 (95\% CI, .18-.77; $P=.0009)$.

Table 4. Preoperative and postoperative VAS and ODI scores.

\begin{tabular}{lccc}
\hline & Pre op & Post op & $\boldsymbol{P}$ Value \\
\hline VAS back pain & $6 \pm 0.7$ & $2 \pm 0.8$ & $P<.0001$ \\
VAS leg pain & $7 \pm 0.4$ & $0.3 \pm 0.1$ & $P<.0001$ \\
ODI & $56 \pm 6.9$ & $18 \pm 4.2$ & $P<.0001$ \\
\hline $\begin{array}{l}\text { Abbreviations: ODI, Oswestry Disability Index; } \\
\text { preoperative; VAS, visual analog scale. }\end{array}$
\end{tabular}
Abbreviations: ODI, Oswestry Disability Index; Post op, postoperative; Pre op,
preoperative; VAS, visual analog scale. 

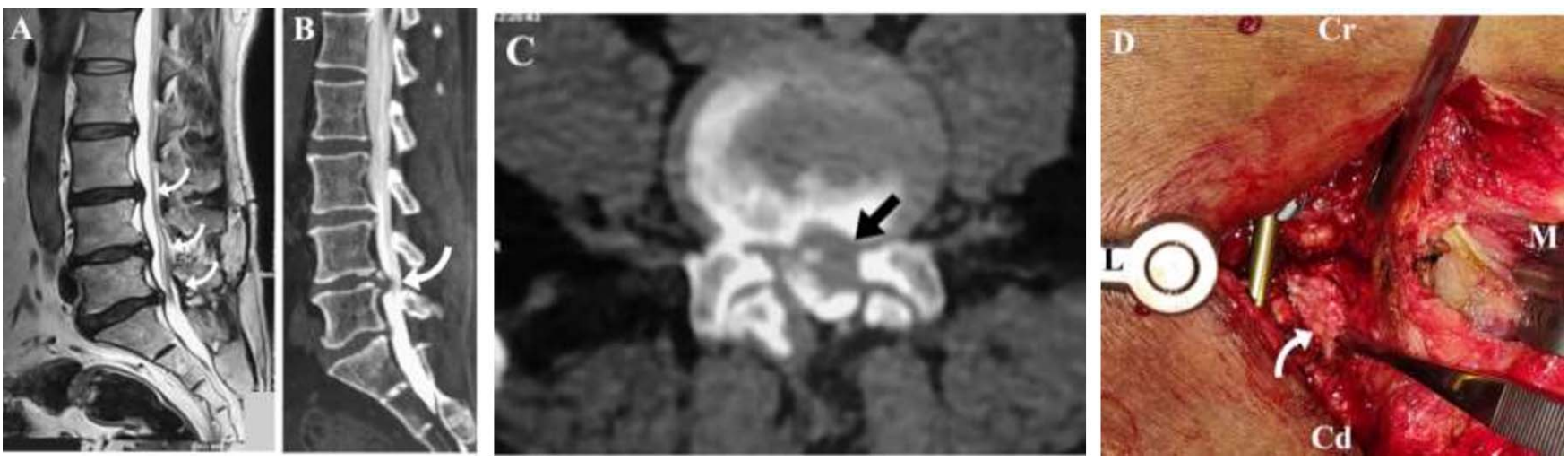

Figure 1. A 60-year-old gentleman presented with low back pain radiating to left lower limb with claudication distance of $100 \mathrm{~m}$ for $3 \mathrm{months}$. (A) Sagittal MRI showing multilevel disc protrusion with predominant compression at L4-L5 and L5-S1 levels. (B) Sagittal computed tomography myelography (CTM) and (C) axial CTM showing left paracentral disc bulge at the L4-L5 level with no other significant compression. (D) Intraoperative image of transforaminal lumbar interbody fusion with leftsided unilateral exposure at the L4-L5 level with disc fragment marked by arrow. Cr, cranial; Cd, caudal; L, lateral; M, medial. At follow-up after 3 months patient had no leg pain and had improved visual analog scale (VAS) scores for back and leg pain as well as improved Oswestry Disability Index (ODI) scores.

In our study, there was no significant adverse reaction post-CTM. Only 1 patient had a transient headache, which subsided in 2 days.

\section{DISCUSSION}

MRI has become the primary diagnostic imaging modality over the last 2 decades for degenerative disorders of the lumbar spine. Despite its efficacy and noninvasive nature, clinicians are confronted

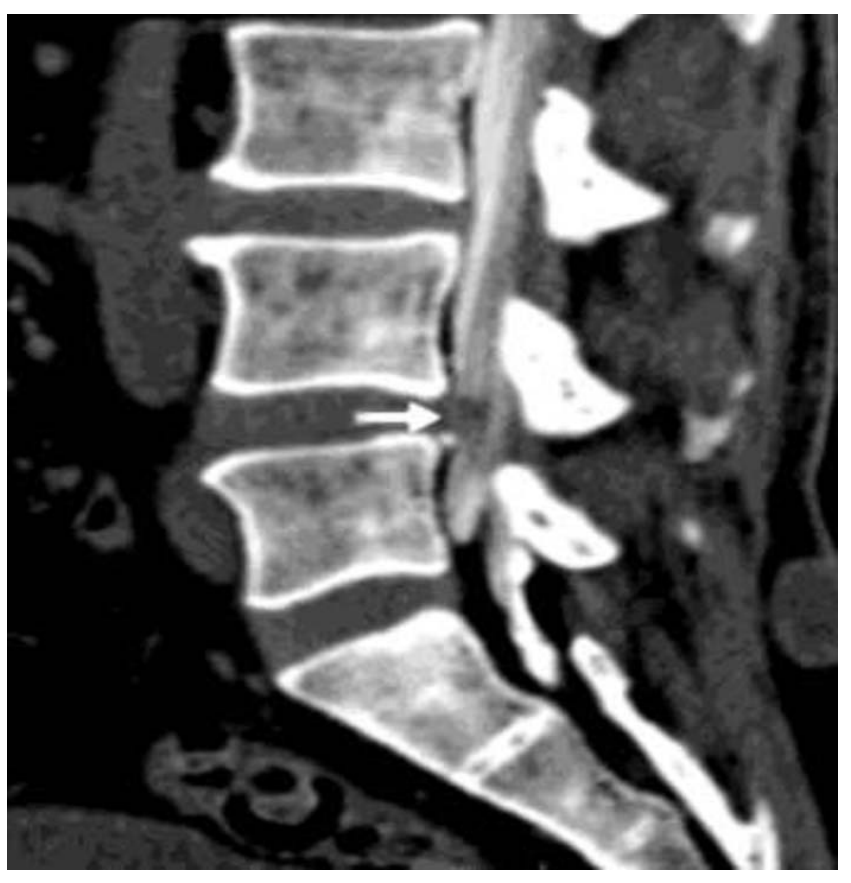

Figure 2. Root impingement. A 63-year-old gentleman presented with low back pain radiating to the left lower limb with a claudication time of 5 minutes for 6 months. MRI did not show any significant compression. Computed tomography myelography (CTM) showed left paracentral disc compressing the traversing nerve root at the L4-L5 level. Transforaminal lumbar interbody fusion (TLIF) L4-L5 was done, and at 6 months follow-up the patient had no radicular symptoms. with management dilemmas when the MRI findings do not correlate with clinical findings in a symptomatic patient or report several levels of significant compression. We report a series of such patients in whom a CTM was performed as an additional imaging modality and was very helpful in surgical planning by clarifying the presence of neural compression by either disc herniation or NDP as well as by accurately localizing the levels of compression. In this series, the levels of decompression were as localized by CTM and all patients reported improvement in their functional status postintervention.

MRI is limited not only in specificity but also in some instances in accurately depicting the pathoanatomic state. CTM is accurate and more specific because of the ability to distinguish bone osteophytes from soft tissue. ${ }^{2-4,6,11}$ MRI is not reliable in delineating compression caused by degenerative changes in the lateral recess, whereas conventional CT and CTM are more accurate when using surgery as the reference standard to confirm degenerative root impingement in the lateral recess as the cause of radiculopathy. ${ }^{6,12}$ In a recent study, Rocos et $\mathrm{al}^{13}$ concluded that CTM has a selective role in delineating central and extraforaminal compression in the lumbar spine.

Advantages of using CTM in conjunction with or in lieu of MRI are improved visualization of the extent of disc herniation, demonstration of focal neural compression by small herniations and extruded discs, clarification of abnormalities of the facets, including synovial cysts, and delineation of osseous pathoanatomy especially in patients with previous surgical history. 


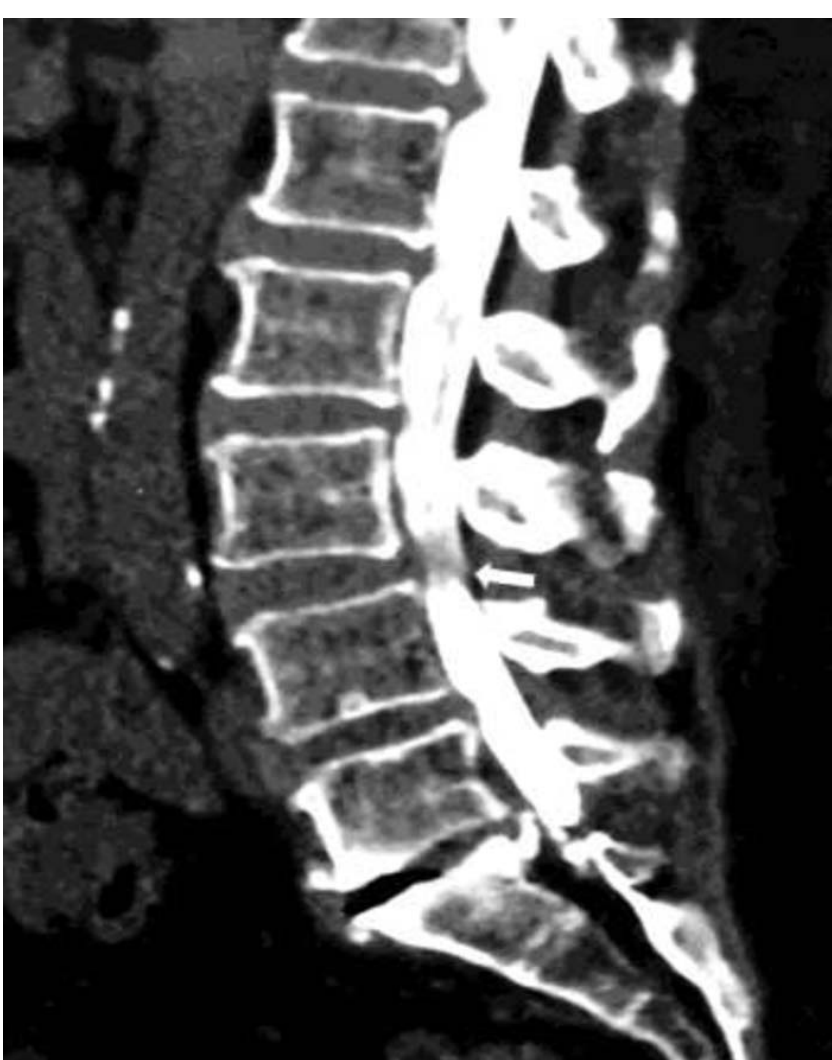

Figure 3. A 66-year-old lady with multiple comorbidities presented with low back pain radiating to both lower limbs (left more than right) with a claudication time of less than 5 minutes. MRI showed compression at multiple levels. Computed tomography myelography (CTM) curved sagittal reconstruction corrected for mild scoliosis and showed major compression at the L3-L4 level. Patient underwent transforaminal lumbar interbody fusion (TLIF) at the L3-L4 level, and at 6 months follow-up she had improved visual analog scale (VAS) and Oswestry Disability Index (ODI) scores. In such cases, multilevel decompression would have added increased surgical morbidity in a frail patient.

The decision for decompression surgery for symptomatic lumbar degenerative disorder largely depends on the severity of stenosis on imaging and clinical findings. However, no universally accepted imaging criteria have been set to define the severity of the lumbar DDD. The cross-sectional spinal canal area has been used as an objective parameter, and an earlier study ${ }^{14}$ revealed that preoperative symptoms of central spinal stenosis are related to the minimum cross-sectional area of subarachnoid space. However, another study ${ }^{15}$ showed that the cross-sectional area varied extensively between patients and the existence of stenosis in a particular patient was difficult to predict.

Our study differs from previous studies on CTM, in that we used thin sections $(0.5 \mathrm{~mm})$ to generate high-resolution multiplanar images. Our curvilinear reconstructions helped assess the compression of the thecal sac more accurately because it aligned to one 2-D longitudinal plane that could be rotated along the centerline through the center of the thecal sac. We also used oblique planar reconstructions that were aligned as true parallel or orthogonal to the plane of the intervertebral discs or the exiting nerve roots, which demonstrated the pathology better (Figure 3). All our patients demonstrated significant improvement in back pain, leg pain, and ODI postsurgery. Our findings indicate that CTM with multiplanar and curvilinear reconstructions provides additional information and helps in better decision-making than MRI alone in cases in which MRI does not correlate with clinical findings.

Sasaki et $\mathrm{al}^{16}$ compared the diagnostic accuracy of MRI, CTM, and myelography in selected patients with lumbar canal stenosis. In their study, sensitivity was $75.9 \%$ for MRI, $87 \%$ for myelography, and $94.4 \%$ for CTM. Their study revealed a significant relationship between surgical findings and myelography/CTM as compared with MRI, concluding that surgical planning on the basis of MRI is less reliable in selected cases. They further showed that CTM was more sensitive than axial MRI and showed compressions in 12 patients that were not detected by axial MRI. Shiban et $\mathrm{al}^{17}$ compared the use of MRI/MRI myelogram with conventional myelography/CTM for detailed surgery planning in degenerative lumbar disease. In their center, surgery was planned on the basis of conventional myelography/CTM. In their series of 26 patients, post hoc surgical planning was repeated based on MRI/magnetic resonance myelogram performed by 6 independent neurosurgeons. Shiban et $\mathrm{al}^{17}$ found that $31 \%(\mathrm{n}=8)$ of the patients had the same surgical decision as originally made, whereas $69 \%(\mathrm{n}=18)$ had different surgical planning as compared with surgery done on the basis of conventional myelography/CTM. Of their patients, $69 \%$ would have had a different surgical procedure if the surgical decision-making was based upon magnetic resonance myelography and not as originally performed, based upon conventional myelography. The authors concluded that surgical planning for the complex lumbar degenerative disorder is highly dependent on the imaging modality used, and discrepancies noted in their series could be because 2 different imaging modalities led to different interpretations.

Morita et al ${ }^{18}$ retrospectively selected 50 patients who underwent decompressive surgery for lumbar spinal canal stenosis and investigated the reliability and reproducibility of MRI and myelography with 


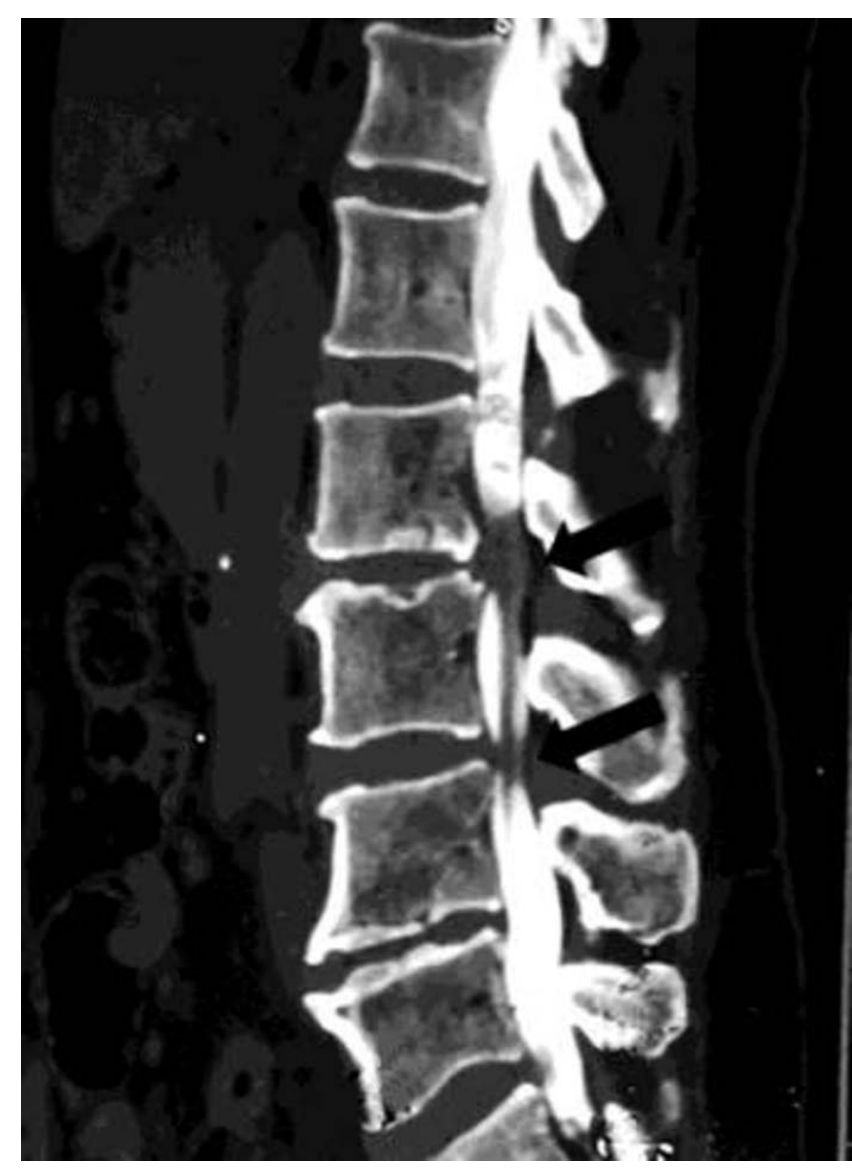

Figure 4. A 70-year-old woman presented with low back pain radiating to the left anterior aspect of the thigh and lateral aspect of the left leg with a claudication distance less than $500 \mathrm{~m}$ for the past 1 year. MRI showed multilevel compression. Computed tomography myelography (CTM) with curved sagittal reconstruction clearly showed canal stenosis at L2-L3 and a left paracentral compression at the L3-L4 levels. The patient underwent transforaminal lumbar interbody fusion (TLIF) at the L2-L3 and L3-L4 levels. At 6 months follow-up, she had no radicular pain and improved visual analog scale (VAS) and Oswestry Disability Index (ODI) scores.

CT in surgical planning of decompressive levels by tasking 4 spine surgeons to decide levels of decompression retrospectively on different occasions based on the findings of MRI or myelography with CTM. They concluded that myelography with CTM is more reliable and reproducible than MRI for preoperative evaluation of lumbar spinal canal stenosis.

In a recent study Morgalla et $\mathrm{al}^{19}$ compared the sensitivity and specificity of 3 imaging modalities for the diagnosis of lumbar spinal stenosis: MRI, CTM, and functional myelography. They reported that functional myelography had the highest sensitivity and specificity, followed by CTM followed by MRI.

They observed that functional myelography revealed the highest precision in reaching a correct diagnosis, and it resulted in a change in the surgical approach in every fifth patient in comparison with the MRI.

In our study, the levels operated upon were decided by CTM findings, and all patients reported statistically significant improvement in their clinical outcome. If we had relied on MRI alone, then we would have subjected patients to more levels of decompression and surgical morbidity with no additional benefit in their functional outcome (Figure 4). Overestimation of MRI is not surprising because prior studies demonstrated that MRI findings of spinal degeneration were prevalent in a large proportion of both symptomatic and asymptomatic individuals, thus limiting the diagnostic value of these findings. ${ }^{20-22}$

Every imaging modality has its own advantages and disadvantages. The disadvantages of CTM include its invasiveness and radiation exposure. MRI remains the first choice of advanced imaging, and CTM can supplement it for surgical decisionmaking when MRI findings do not correlate with clinical findings and especially when the patient has multiple segment degeneration.

However, some limitations that may have biased the study findings must be considered. We have not done decompression at levels in which MRI showed compression, and hence we cannot confirm whether compression was present. However, postoperative favorable outcome on follow-up confirms that there was no significant compression at these levels contributing to patient symptoms. We also have not considered measurable objective parameters evaluating the severity of lumbar degenerative diseases such as cross-sectional area because our intention in this pragmatic study was to correlate clinical and radiological tools for decision-making. In this series, we examined only static CTM studies comparable with recumbent MRI. Future work should include dynamic studies in their analysis.

\section{CONCLUSIONS}

CTM is an adjunct imaging study that is necessary in patients presenting with symptomatic lumbar DDD to confirm degenerative impingement in cases in which MRI does not reveal compressive pathology or shows multilevel changes. Our experience shows that CTM is an accurate and reliable modality to identify the significant stenotic levels and offers a good outcome when the management plan is based on it. 


\section{REFERENCES}

1. Franken EA, Berbaum KS, Dunn V, et al. Impact of MR imaging on clinical diagnosis and management: a prospective study. Radiology. 1986;161(2):377-380.

2. Haughton VM. MR imaging of the spine. Radiology. 1988;166(2):297-301.

3. Lee HS, Coleman PE, Hahn FJ. Magnetic resonance imaging of degenerative disk disease of the spine. Radiol Clin North Am. 1988;26(5):949-964.

4. Modic MT, Masaryk T. Paushter D. Magnetic resonance imaging of the spine. Radiol Clin North Am. 1986;24(2):229245 .

5. Ortiz O, Pait TG, McAllister P, Sauter K. Postoperative magnetic resonance imaging with titanium implants of the thoracic and lumbar spine. Neurosurgery. 1996;38(4):741-745.

6. Goldberg A, Soo MS, Deeb ZL, et al. Degenerative disease of lumbar spine: role of CT-myelography in the MR era. Clin Imaging. 1991;15(1):47-55.

7. Boonstra AM, Schiphorst Preuper HR, Reneman M, Posthumus JB, Stewart RE. Reliability and validity of the visual analogue scale for disability in patients with chronic musculoskeletal pain. Int J Rehabil Res. 2008;31(2):165-169.

8. Fairbank J, Pynsent P. The Oswestry Disability Index. Spine. 2000;25(22):2940-2953.

9. Kendrick R, ed. Social Statistics: An Introduction Using SPSS for Windows. Boston: Allyn \& Bacon; 2005.

10. Landis $\mathrm{J}$, Koch $\mathrm{G}$. The measurement of observer agreement for categorical data. Biometrics. 1977;33(1):159-174.

11. Jia LS, Shi ZR. MRI and myelography in the diagnosis of lumbar canal stenosis and disc herniation. A comparative study. Chin Med J. 1991;104(4):303-306.

12. Nielsen JA, Carrino JA. Computed tomography. In: Waldman SD, ed. Pain Management. Philadelphia, PA: Saunders, 2007:93-105.

13. Roco B, Evans DRS, Rajayogeswaran B, et al. The normal appearance of CT myelograms. Eur Spine $J$. 2020;29(5):1087-1091. doi:10.1007/s00586-019-06287-5

14. Ogikubo O, Forsberg L, Hansson T. The relationship between the cross-sectional area of the cauda equine and the preoperative symptoms in central lumbar spinal stenosis. Spine. 2007;32(13):1423-1428.

15. Speciale AC, Pietrobon R, Urban CW, et al. Observer variability in assessing lumbar spinal stenosis severity on magnetic resonance imaging and its relation to cross-sectional spinal canal area. Spine. 2002;27(10):1082-1086.
16. Sasaki K, Hasegawa K, Shimoda H, Keiji I, Homma T. Can recumbent magnetic resonance imaging replace myelography or computed tomography myelography for detecting lumbar spinal stenosis? Eur J Orthop Surg Traumatol. 2013;23(suppl 1):S77-S83.

17. Shiban E, von Lehe M, Simon M, et al. Evaluation of degenerative disease of the lumbar spine: MR/MR myelography versus conventional myelography/post-myelography CT. Acta Neurochir (Wien). 2016;158(8):1571-1578.

18. Morita M, Miyauchi A, Okuda S, Oda T, Iwasaki M. Comparison between MRI and myelography in lumbar spinal canal stenosis for the decision of levels of decompression surgery. Clin Spine Surg. 2011;24(1):31-36.

19. Morgalla M, Frantz S, Dezena RA, Pereira CU, Tatagiba M. Diagnosis of lumbar spinal stenosis with functional myelography. J Neurol Surg A Cent Eur Neurosurg. 2018;79(4):316-322.

20. Boden SD, Davis DO, Dina TS, et al. Abnormal magnetic-resonance scans of the lumbar spine in asymptomatic subjects: a prospective investigation. J Bone Joint Surg Am. 1990;72(3):403-408.

21. Jensen MC, Brant-Zawadzki MN, Obuchowski N, et al. Magnetic resonance imaging of the lumbar spine in people without back pain. $N$ Engl J Med. 1994;331(2):69-73.

22. Matsumoto M, Okada E, Toyama Y, et al. Tandem agerelated lumbar and cervical intervertebral disc changes in asymptomatic subjects. Eur Spine J. 2013;22(4):708-713.

Disclosures and COI: The authors received no funding for this study and report no conflicts of interest.

Corresponding Author: Dr Keyur K. Akbari, Spine Unit, Apollo Hospital, Greams Lane, Chennai 600006 , India. Phone: +918980808179 or +919879137187; Email: keyur21088@yahoo.co.in

Published 19 August 2021

This manuscript is generously published free of charge by ISASS, the International Society for the Advancement of Spine Surgery. Copyright (C) 2021 ISASS. To see more or order reprints or permissions, see http://ijssurgery.com. 